\title{
Formal Development of Basic Timestamp Concurrency Control Mechanism using Event-B
}

\author{
Girish Chandra \\ Computer Science \& Engineering Department \\ Institute of Engineering \& Technology \\ Lucknow, India
}

\author{
Divakar Yadav \\ Computer Science \& Engineering Department \\ Institute of Engineering \& Technology \\ Lucknow, India
}

\begin{abstract}
Formal methods are mathematical techniques that are used to develop model of complex systems. They provide mathematical proofs for ensuring correctness of model. Through formal methods, it may possible to identify and remove errors at prior stage of development. Event-B is a formal method that is used to develop those systems that can be modeled as discrete transition systems. It rigorously describes the problem and verifies the correctness of model by discharging proof obligations. It performs the consistency checking by preserving invariants of model. In this paper, we have done formal verification of basic time stamp mechanism using Event-B. Basic time stamp is concurrency control mechanism to control concurrent execution of transactions. The main objective of timestamp is to execute transactions such that their execution is equivalent to serial execution in time stamp order.
\end{abstract}

\section{Keywords}

Formal Methods; Formal Specifications; Event-B; Database; Transaction; Basic Timestamp Mechanism.

\section{INTRODUCTION}

The tremendous growth of complex system has developed research interest to build system which is free from failures. Due to faulty specification, it may possible that developed system may involve errors that may cause failure of the system. For safety critical system, fault tolerance and reliability are the main features. Therefore, it is highly recommended that system must capture correct specification and it should be rigorously verified during its development. The size of state space is very large for complex system. It is unfeasible to correctly verify every execution path for such system using traditional testing methods.

Formal methods are mathematical techniques that are used for modelling and verification of complex systems [1]. They provide mathematical proofs for ensuring correctness of system. Through formal specifications, the system can be correctly verified at the design stage of the development [2]. Formal specifications are well defined mathematical semantics whose purpose is to model the correct system by formalizing all system requirements while hiding implementation details [3]. Event-B [3] [4] [5] is a formal method that is used to develop those systems that can be modeled as discrete transition systems. Modeling through Event-B supports stepwise development of model. It verifies abstract specification and adds details in refinement steps in order to obtain concrete specifications [4].

In distributed database system, scheduler at each site is responsible for managing concurrent access to data items stored at that site [6]. Concurrency control is activity of controlling concurrent execution of transaction such that data consistency is maintained [7]. Serial execution of transactions provides high consistency to database but it losses the efficiency of system. In serial execution, it may possible that transaction has to wait for longer period of time [6]. Therefore, parallel execution of transactions is preferred. The main difficulty during parallel execution of transaction is how to maintain the consistency. For achieving it, concurrency control synchronizes the concurrent execution of transaction by preventing the modification of data objects when any transaction is accessing it. Synchronization of transaction execution is done either by applying the locks or by using timestamp techniques [7]. The lock based techniques ensures serializability by applying various modes of locks on required data objects. The timestamp based techniques use timestamp ordering to determine the order between every pair of conflicting transactions [7] [8]. In this technique, when a transaction is submitted in the system a unique timestamp is assigned to it. The timestamp of transactions decide the serializability order. For example, if timestamp of any transaction $T a$ is less than timestamp of other transaction $T b$ then the system must ensure that produce scheduled must be equivalent to a serial schedule where transaction $T a$ appears before $T b$. The timestamp based techniques can be further categorized [7] as basic timestamp based approach, conservative approach and multi-version concurrency control system. We have done formal modeling of multi-version concurrency control system in [9].

In this paper, we have considered basic timestamp based concurrency control [7] for formal development of our model. In this approach, transaction will perform successfully read and write operation on data items if they are not written by some younger transactions. We have done formal verification of basic timestamp mechanism using Event-B as a formal method. The remainder of this paper is organised as follows: section II briefly outline our modelling approach, section III describes informal description about our model and events, section IV presents formal specifications of basic timestamp mechanism. Finally, section V concludes our paper.

\section{EVENT-B}

Event-B [2][3][4][5][10][11] is an event driven based formal method which is an extension of classical B method. It is used to formalize and develop those systems that can be modeled as discrete transition systems. Event-B model contains two basic construct of two types [12] [13]: contexts and machines. Context represents static part of model. It may contain the declaration of sets, constants and axioms. Depending on the requirement set may be carrier set or enumerated set. Axioms are used to represent properties of sets. On the other hand, 
A mapping (dimdv) $\in$ database represents that database consists data item $d i$ having its value $d v$. The variable transactiontsp is declared as total function from each started transaction to natural number. It represents timestamp of transaction. Whenever, a new transaction is submitted this natural number is incremented by one. The declaration of other variables are as follows:

- The variable activetrans is declared as subset of started transactions. It represents set of active transactions for which conflict checking has been done and data items are available.

- The variable transdataitem is declared as:

$$
\text { transdataitem } \in \text { transaction } \rightarrow \text { Pow } 1 \text { (DATAITEM) }
$$

It represents set of dataitem required by transaction. A mapping of form (trmDI) $\in$ transdataitem represents that transaction $t r$ needs data items $D I$.

- The variable writetsp represents write timestamp of data item.

- Similarly, variable readtsp represents read timestamp of data item.

The variable transstatus maps each submitted transactions to one of its status. At any instance, transaction may be in following state: PENDING, ACTIVE, READCOMMIT, WRITECOMMIT and ABORT.

Submit_Transaction $\hat{=}$
Any $t r$,dataitem Where
grd1: $t r:$ TRANSACTION
grd2: $t r$ / transaction
grd3: dataitem $:$ Pow1(DATAITEM)
Then
act1: transaction $:=$ transaction $U\{t r\}$
act2: transactiontsp $($ tr $):=$ counter
act3: counter $:=$ counter +1
act4: transdataitem $($ tr $):=$ dataitem
act5: transstatus $($ tr $):=$ PENDING
F.nd

Fig. 2. Submit_Transaction Event

Conflict_Checking $\triangleq$
Any $t r$ Where
grd1: $t r:$ transaction
grd2: $t r /$ activetrans
grd3: transstatus $($ tr $)=P E N D I N G$
grd4: $\forall$ tt $\cdot(t t \in$ activetrans $\Rightarrow$
Then
act1: transstatustem $(t r) \cap$ transdataitem $(t t)=\emptyset)$
act2: activetrans $:=$ activetrans $U\{t r\}$
End

Fig. 3. Conflict_Checking Event

\subsection{Submit_Transaction Event}

This event models the submission of transaction (Fig. 2). The guard grd1 and grd2 ensure that transaction tr is fresh transaction. The dataitem is a set which contain all data items required by transaction $t r$. Each time when a transaction is submitted a unique timestamp is allotted to it (act2). The action act4 specifies that dataitem are assigned to transaction $t r$. The status of transaction $t r$ is set to as PENDING through act5.

\subsection{Conflict_Checking Event}

Data items required by the submitted transaction may be locked by other transactions. This event formalizes conflict checking process (Fig. 3). The guard grd2 specifies that transaction $t r$ is not active transaction. The guard $g r 3$ ensures that status of transaction $t r$ is PENDING. The guard $g r d 4$ is written as:

$\forall t t \cdot(t t \in$ activetrans $G$ transdataitem $(t r) \cap \operatorname{transdataitem}(t t)=\varnothing)$

It ensures that data items required by transaction $t r$ are not conflict with data items used by other active transactions $t t$ i.e. (transdataitem $(t r) \cap$ transdataitem $(t t)$ ) will be equal to $\emptyset$. This event sets the status of transaction $\operatorname{tr}$ as ACTIVE.

\subsection{Read_Operation Event}

Read_Operation event is given in Fig. 4. The transaction will perform successfully read operation on data item if write timestamp of data item will be less than transaction timestamp

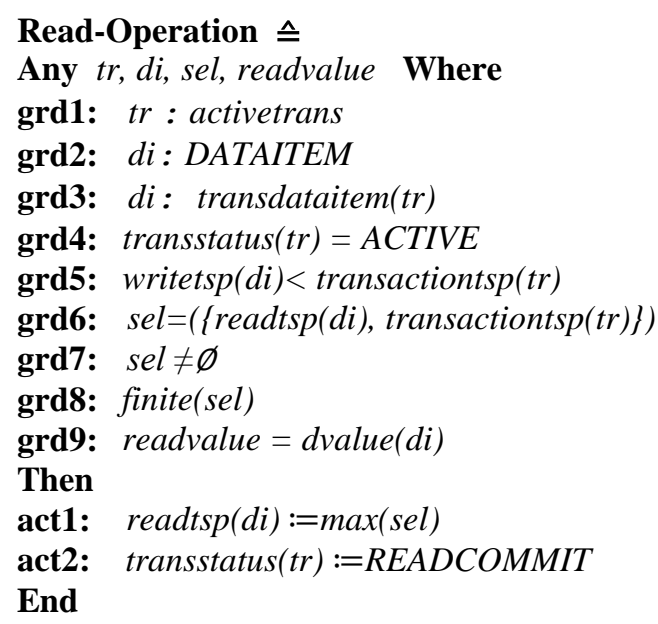

Fig. 4. Read_Operation Event

The guard $g r d 3$ represents that transaction $t r$ wants to read data item $d i$. The status of transaction $t r$ is ACTIVE is ensured by guard grd4. The write timestamp of data item $d i$ is less than transaction's timestamp $t r$ is ensured through $g r d 5$. The guard grd6 is written as:

$$
\text { sel }=(\{\text { readtsp }(\text { di }), \text { transactiontsp }(\text { tr })\})
$$

It represents a set sel which contains read timestamp of data item $d i$ and timestamp of transaction $t r$. The guard grd 9 returns the value of data item $d i$. The occurrence of this event updates the read timestamp of data item $d i$. The new read timestamp of data item $d i$ will be maximum value of current read timestamp of data item $d i$ and timestamp of transaction $t r$ (act1). The action act2 sets the status of transaction $t r$ as READCOMMIT

\subsection{Write_Operation Event}

This event formalizes update operation (see Fig. 5). Any active transaction may perform successfully write operation over data item if transaction's timestamp will be greater than 
read and write time stamp of data item. The guard $\operatorname{grd} 7$ and grd8 ensure that write and read timestamp of data item $d i$ are less than transaction's timestamp $t r$, respectively. This event updates data value $d i(a c t 1)$. It also updates write timestamp of data item $d i$ as transaction timestamp $\operatorname{tr}($ act2) and set the status of transaction $t r$ as WRITECOMMIT (act3).

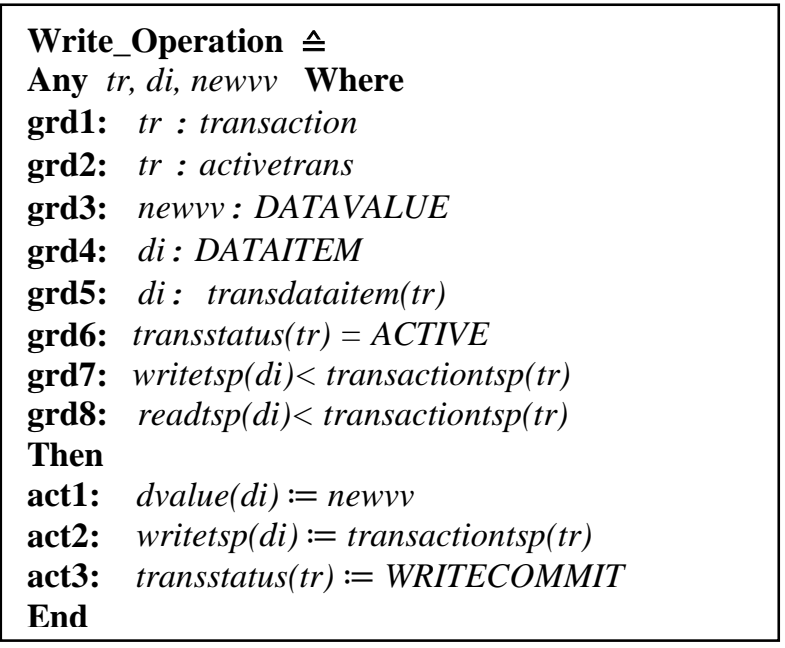

Fig. 5. Write_Operation Event

Read_Write_Abort $\triangleq$
Any $t r$, d $i$ Where
grd1: $t r:$ transaction
grd2: tr $:$ activetrans
grd3: $d i:$ DATAITEM
grd4: di $:$ transdataitem $(t r)$
grd5: transstatus $($ tr $)=A C T I V E$
grd6: writetsp $($ di $)>$ transactiontsp $(t r)$
Then
act1: transstatus $($ tr $):=A B O R T$
act2: activetrans $:=$ activetrans $\backslash\{\operatorname{tr}\}$
End

Fig. 6. Read_Write_Abort Event

Write_Abort $\hat{=}$
Any $t r, d i$ Where
grd1: $t r:$ transaction
grd2: $t r:$ activetrans
grd3: $d i:$ DATAITEM
grd4: di $:$ transdataitem $(t r)$
grd5: transstatus $($ tr $)=$ ACTIVE
grd6: readtsp $($ di $)>$ transactiontsp $($ tr $)$
Then
act1: transstatus $($ tr $):=$ ABORT
act2: activetrans $:=$ activetrans $\backslash\{\operatorname{tr}\}$
End

Fig. 7. Write_Abort Event

\subsection{Read_Write_Abort Event}

The abortion of any read and write operation will occur if transaction does not succeed either in reading or writing on data item. It will be possible if write timestamp of data item will greater than transaction's timestamp. The guard grd6 of
Fig. 6. Indicates that write timestamp of data item $d i$ is greater than transaction's timestamp tr. Therefore, transaction any of its kind either update transaction or read only transaction will be aborted.

\subsection{Write_Abort Event}

The event Write_Abort is given in Fig. 7. The write operation will also be aborted if required data items are already been read by some younger transaction. The guard grd6 indicates that data item di is already read by younger transaction because read timestamp of data item $d i$ is greater than transaction's timestamp $\operatorname{tr}$ ( $g r d 6$ ). The action actl changes the status of transaction $\operatorname{tr}$ as $A B O R T$.

\section{CONCLUSION}

The concurrency control can be provided either through locks or timestamps. The timestamp based approaches are categorized as basic timestamp mechanism, conservative approach and multiversion approach. Formal modeling of multiversion concurrency control system using Event-B can be found in [9]. In this paper, we have done formal modeling of basic timestamp mechanism using Event-B. The basic timestamp mechanism ensures that execution of transaction is equivalent to serial execution in timestamp order. In this approach, a transaction will perform read and write operation on any data item if that data item had been last written by an older transaction. For ensuring correctness of system, it is required to verify every execution path of model.

Formal methods are mathematical techniques which provide systematic approach for development and reasoning about complex system. They provide proof based approach to verify the correctness of model. We have considered Event-B formal method for development of our model. We have done verification by preserving invariants. Invariants are constraints on machine variables. During execution of model these invariants should not be violated. Invariant preservation is ensured by discharging proof obligations generated by systems. We have used Rodin platform for formalizing our model. Total 43 proof obligations are generated by system out of which 29 proofs are discharged by the prover of Rodin tool automatically while 14 proofs are discharged interactively. While discharging the proof obligations, it gives clear reasoning about the model. In future, we are planning to formalize conservative approach of timestamp in distributed environment

\section{REFERENCES}

[1] Butler, M. and Maamria, I.. Practical theory extension in Event-B. In Zhiming Liu, Jim Woodcock, and Huibiao Zhu, editors, Theories of Programming and Formal Methods, volume 8051 of Lecture Notes in Computer Science, pages 6781. Springer, 2013.

[2] Hallerstede, S. and Leuschel, M.: Experiments in program verification using Event-B. Formal Aspects of Computing, 24: pp. 97125, (2012).

[3] Yadav D. and Butler M.: Application of Event B to Global Causal Ordering for Fault Tolerant Transactions. In: Proc. of REFT 2005, Newcastle upon Tyne, pp. 93 103, (2005).

[4] Butler, M.and Yadav D.: An incremental development of the mondex system in Event-B. Formal Aspects of Computing, 20(1):61-77, (2008). 
[5] Banach R.: Retrenchment for Event-B: UseCase-wise development and Rodin integration. Formal Aspects of Computing, 23, pp. 113131, (2011).

[6] Ozsu M. and Valduriez P.: Principles of Distributed Database Systems Pearson Education (Singapore) Pte.Ltd. India (2004).

[7] Bernstein, P. and Goodman, N.: Timestamp Based Algorithms for Concurrency Control in Distributed Database Systems. In: Proc. of 6th Int. Conf. on Very Large Databases (1980).

[8] Bernstein, P., Hadzilacos, V. and Goodman, N.: Concurrency Control and Recovery in Database Systems. Addison-Wesley (1987).

[9] Suryavanshi, R. and Yadav, D. "Modeling of Multiversion Concurrency Control System Using EventB" in Federated Conference on Computer Science and Information systems (FedCSIS), indexed and published by IEEE, 9-12 September, Wroclaw, Poland, 2012.

[10] Suryavanshi, R. and Yadav, D. :Formal Development of Byzantine Immune Total Order Broadcast System using Event-B. In: ICDEM 2010, F. Andres and R. Kannan (eds.) LNCS, Vol. 6411, Springer, pp.317-324, (2010).

[11] Yadav, D. and Butler, M.: Formal Development of a Total Order Broadcast for Distributed Transactions
Using Event-B. Lecture Notes in Computer Science 5454, springer-Verlag Berlin Heidelberg, pp.152-176, (2009).

[12] Basin, D., Furst, A., Hoang, T.S., Miyazaki, K. and Sato, N. Abstract Data Types in Event-B - An Application of Generic Instantiation. CoRR, 2012.

[13] Metayer, C., Abrial, J R. and Voison L.: Event-B language. RODIN deliverables 3.2, http://rodin.cs.ncl.ac.uk/deliverables/D7.pdf, (2005).

[14] Hallerstede, S.: On the purpose of Event-B proof obligations. FormalAspects of Computing, 23: pp. 133150, (2011).

[15] Abrial, J-R. From $Z$ to B and then Event-B: Assigning Proofs to Meaningful Programs. In E.B. Johnsen and L. Petre, editors, IFM, volume 7940 of Lecture Notes in Computer Science, pages 115. Springer, 2013.

[16] Banach, R.: Retrenchment for Event-B: UseCase-wise development and Rodin integration. Formal Aspects of Computing, 23, pp. 113131, (2011).

[17] Abrial, J R.: A system development process with Event$\mathrm{B}$ and the Rodin platform. In: Lecture Notes In Computer Science 4789, Springer, pp.1-3,(2007). 